\title{
Fundamental Diagram Modelling and Analysis Based NGSIM Data
}

\author{
X. Y. Lu*, P. Varaiya**, and R. Horowitz *** \\ *PATH, U. C., Berkeley, Richmond Field Station, Bldg 4521357 S. 46th Street, Richmond, \\ CA 94804-4648, USA (Tel:510-665 3644; e-mail: xylu@path.berkeley.edu). \\ ** EECS, U. C. Berkeley, 271M Cory Hall, Berkeley CA 94720-1770 \\ USA (e-mail: variya@eecs.berkeley.edu) \\ *** ME, U. C. Berkeley, 5138 Etcheverry Hall, Berkeley CA 94720-1770 \\ USA (e-mail: horowitz@me.berkeley.edu)
}

\begin{abstract}
Traffic control requires looking at traffic models of all types in finer details. This paper is to investigate lane-wise flow-density (or equivalently speed-density) relationship which is traditionally called Fundamental Diagram (FD) over a stretch of homogeneous freeway section using the microscopic NGSIM data. Particularly, it investigates how a homogenous traffic further drop (breakdown) through data analysis and modeling. The breakdown of a homogenous traffic is understood as the significant flow drop and density increase with noticeable shock-wave back-propagation. The corresponding density is a generalization of the critical density for traffic breakdown from free-flow. Variable structure models with two limbs of the inverse $\lambda$-shape are proposed to model the homogenous flow and its further drop. A special Generalized Polynomial Model (with fraction coefficients) is also proposed for the right limb. Properly aggregated NGSIM data are used to fit the model with results compared with some other models over time at fixed location using Root Mean Square Errors (RMSE) as measure. Principles for time/distance aggregation of individual vehicle trajectories to macroscopic traffic state parameters are proposed.
\end{abstract}

Keywords: Fundamental Diagram, variable structure modeling, speed-density relationship, equilibriums state, homogenous flow, NGSIM data 


\section{INTRODUCTION}

In the nominal work of Greenshields (1934), the Fundament Diagram (FD) was defined and used as the relationship between traffic flow $q$ and density $\rho$ for an equilibrium traffic state. Sine then, several works have been conducted to establish a static relationship between flow and density in theory and in empirical modelling with filed data fitting. It is generally recognized that FD is location dependent due to road geometry and traffic characteristics. FD may have several equivalent forms: flow-density (occupancy) which is concave, speed-density (occupancy) which is monotone decreasing, and speedflow with two foliations: upper limb and lower limb. It is noted that the speed- $u / c$ (volume/capacity ratio) relationship in HCM (Highway Capacity Manual) is equivalent to a speed-flow relationship. Data aggregation level in time for modelling determines the application of the FD model: short time aggregated data leads to model for traffic operation, and long time aggregated data lead to model for planning (Skabardonis, 1977). Although some models are for planning purpose, some are for operation, and several models could be used for both purpose depending on the time aggregation level. In fact, several models calibrated on the same set of field data could produce the similar outputs for the same input data with similar level of model mismatch error such as Mean Square Error (MSE). A good model should be flexible enough to capture the intrinsic functional relationship for a range of field data.

Lighthill and Whitman (1995) investigated the FD in $q-\rho$ (flow-density) plane and suggested that the FD should have a flat top as depicted by Greenshields (1934). Del Castillo et al (1995) considered the functional form of the speed-density relationship. The FD can be obtained from $q=v \cdot \rho$ which is true if $v$ is distance means speed and if the data aggregation level for the three are the same.

The FD is the basis of several traffic flow modelling approaches. A generally accepted model is the macroscopic LWR model (Lighthill and Whitham, 1955) which has been used for traffic simulation and control. For practical calculation, the model is usually simplified based on the assumption of the existence of an FD. Thus the speed is eliminated from the LWR model with the density left as the only traffic state variable for the dynamic system, as in the Cell Transmission Model (CTM) (Daganzo, 1994, 1995). A primary result of that paper is that the CTM is equivalent to the LWR hydrodynamic model based the existence of the FD and the assumption of homogeneous highway traffic. For non-homogeneous highway traffic (phase transition), the equivalence would be difficult to prove.

Beside the importance for model simplification, FD is also used to estimate some critical traffic parameters such as capacity/critical flow, critical/jammed density, etc. provided that the FD truly reflects the intrinsic traffic characteristics.
Controversial opinions regarding the existence and forms of the FD are well-known. Different forms of FD have been suggested: (a) the simplest form - a triangle; (b) reversed parabolic and reversed Gambell; and (c) a reversed $\lambda$ etc. However, it is generally agreed that $q=q(\rho)$, if it exists, is a concave function of density $\rho$ defined in $\left[0, \rho_{J}\right] \quad\left(\rho_{J}-j a m\right.$ density $)$ and boundary conditions $q(0)=q\left(\rho_{J}\right)=0 ; \quad$ and the corresponding speed-density relationship is monotone decreasing.

Critical density $\rho_{c}$ is originally defined as the density at which free-flow traffic is to breakdown. It is well-known that traffic may have infinite number of equilibrium state which can be described as homogeneous flow sustainable for certain period of time with mean speed within the interval $\left(0, v_{f}\right]\left(v_{f}\right.$ - free-flow speed $)$, in which speed and density (thus flow) are close to some constants. However, the traffic could further breakdown (or transition) from one equilibrium state to another with lower flow. An strong evidence for this is the shockwave observed through NGSIM data which are collected in peak hours ( $\mathrm{Lu}$ and Skabardonis, 2007). Traffic breakdown from free-flow is to transit from free-flow (a special homogenous flow) to a congested flow. The objective of active traffic control is to achieve the following in the order of priority and feasibility: (i) to smoothly transit to equilibrium state with higher flow; (ii) to keep it a homogenous flow (without shockwave); (iii) to smoothly transit to a homogeneous traffic with lower flow if it is unavoidable (without shockwave). For this purpose, it is not only necessary to understand how the traffic transit (breakdown) to a equilibrium state with lower flow, but also necessary to understand the mechanism and characteristics how to transit smoothly from one equilibrium state to another. The break-down density is denoted as $\rho_{c}^{h}$ for homogenous traffic state $h$, which is understood as the density with abrupt flow drop and density increase, such as, with shockwave back-propagation.

The most accepted FD model from a practical traffic data viewpoint is a reversed $\lambda$ shape with the two limbs, which has also been observed in NGSIM data analysis. The left limb of the FD corresponds to the homogenous-flow (freeflow as a special case) which is close to a straight line and it matches pretty well with practical data. However, different ideas arise as to what shape the right limb corresponding to congested traffic should look like. Notice the following facts: (a) practical data are scattered for the congested flow as shown by many studies; (b) jammed density is fixed as generally accepted, for example, $\rho_{J}=230$ vehicle per lane per mile (vplpm), at which $v\left(\rho_{J}\right)=0$ and $q\left(\rho_{J}\right)=0$ must hold; and (c) the other end of the right limb has to intersect with the left limb at critical density and maximum flow. Usually, the scattered data do not fit the right limb well with those constraints if the model is not properly chosen. To solve the problem, 
this paper proposes several choices for modelling the right limb.

The main contributions of the paper are: (a) A Generalized Polynomial Model with some non-integer coefficients is proposed for $v-\rho$ model, which produce concave $q-\rho$ relationship; Variable structure model is proposed for the reversed $\lambda$ shape FD for homogenous traffic flow. The left limb includes linear and parabolic curves, and the right limb includes linear, Generalized Polynomial, and Edie models; (b) Those variable structure models are compared using the properly aggregated NGSIM data for both $v-\rho$ and $q-\rho$ relationships using Root-Means- Square Error (RMSE) as the performance measure; (c) The density range for further breakdown of the homogenous traffic recorded in NGSIM $\rho_{c}^{H}$ is investigated with the available data sets. It shows that the range is $[90,120]$ per-veh-per-mile for the recorded saturated traffic; this is might be significantly higher than the generally believed critical density range for the free-flow to breakdown; ; and (d) The impact of the value for $\rho_{c}^{h}$ in the range on modelling is investigated with RMSE for comparison of different model combinations. It shows that this value is different, though within the range, in times as well as in locations for the same model.

It is noted that Edie (1961) also suggested using a variable structure model to represent the traffic breakdown near maxim density $\rho_{m}$, where from field data, $\rho_{m} \in[75,100]$ which was close to what is observed with NGSIM data.

The paper is structured as follows: Section 2 review previous work on Fundamental Diagram modelling and application; Section 3 proposes a Generalized Polynomial Model and investigate the temporal behaviour of several FD models for the same location using properly aggregated NGSIM data; Section 4 establishes some variable structure models which are calibrated using properly aggregated NGSIN data. Section 5 is for concluding remarks.

\section{LITERATURE REVEW}

Many models exist for modelling the static $v-\rho$ or $q-\rho$ relationship. Although the function expressions are different, they are more or less similar in the domain $\rho \in\left[0, \rho_{J}\right]$. However, some of them do not satisfy the two boundary conditions $v(0)=v_{f},\left(\rho_{J}\right)=0$ simultaneously.

\subsection{Models for Speed-Density $(v-\rho)$ relationship}

(1) Edie Model

Edie (1961) Showed that the Greenberg model (1959)

$$
v=V_{m} \ln \left(\frac{\rho}{\rho_{J}}\right)
$$

can be obtained by integration of the following carfollowing model

$$
M \dot{x}_{n+1}(t)=\lambda_{1} \frac{\left(\dot{x}_{n}(t+\Delta t)-\dot{x}_{n+1}(t+\Delta t)\right)}{\left(x_{n}(t+\Delta t)-x_{n+1}(t+\Delta t)\right)}
$$

$M-$ vehicle mass

$\lambda_{1}$ - driver sensitivity character coefficient

$\Delta t$ - average time lag, a constant, for driver-car system $x_{n}(t), x_{n+1}(t)$ - the coordinate of front vehicle and the subjective vehicle with respect to the an inertia coordinate system at time $t$. $x_{n}(t)-x_{n+1}(t)$ is the distance headway. It is noted that a constant $\Delta t$ would not affect the integration. Using the relationship between average distance headway and density $\rho=1 / y=\frac{1}{x_{n}-x_{n+1}}$, the boundary condition $v\left(\rho_{J}\right)=0$ and $V_{m}=\lambda_{1} / M,(2.1)$ is obtained. General Greenberg model is obtained by adding parameters for data fitting flexibility:

$$
v(\rho)=g_{1}+g_{2} \ln \left(\frac{\rho}{\rho_{J}}\right)
$$

It is pointed out the flaw of the Greenberg model is

$$
\lim _{\rho \rightarrow 0} V_{m} \ln \left(\frac{\rho_{J}}{\rho}\right)=\infty
$$

which means the model is not suitable for sparse traffic. Edie suggested a further improvement by starting the following microscopic car-following model for the uncongested traffic

$$
M \dot{x}_{n+1}(t)=\lambda_{1} \dot{x}_{n+1}(t) \frac{\left(\dot{x}_{n}(t+\Delta t)-\dot{x}_{n+1}(t+\Delta t)\right)}{\left(x_{n}(t+\Delta t)-x_{n+1}(t+\Delta t)\right)^{2}}
$$

with the boundary condition

$$
v(0)=v_{f} \text { (free-flow) if } \frac{1}{x_{n}-x_{n+1}}=\frac{1}{y}=0 \text { or infinite spacing }
$$

One can reach the following model noticing the density and headway relationship $\rho=1 / y$ :

Or equivalently

$$
\begin{aligned}
& \rho=\rho_{m} \ln \left(\frac{v_{f}}{v}\right) \\
& \rho_{m}=1 / y_{m}
\end{aligned}
$$

$$
v=v_{f} \exp \left(\frac{-\rho}{\rho_{m}}\right)
$$

which is exactly the Underwood model (1961). $y_{m}$ - the spacing of maximum flow which is deduced by minimizing the $q(v) ; \rho_{m}$ - the density of maximum flow. Parameters $w_{1}$ and $w_{2}$ are added for flexibility in data fitting:

$$
v=V(\rho)=\exp \left(-w_{1} \frac{\rho}{\rho_{J}}+w_{2}\right)
$$


It is strictly concave for $\rho \in\left[0,2 \rho_{m}\right)$.

Those two can be combined in one model as:

$$
v(\rho)= \begin{cases}\exp \left(-w_{1} \frac{\rho}{\rho_{J}}+w_{2}\right), & \rho \leq \rho_{J} \\ g_{1}+g_{2} \ln \left(\frac{\rho}{\rho_{J}}\right), & \rho>\rho_{J}\end{cases}
$$

(2) The following polynomial model is cited in (Zhang, 1999) as the one-parameter polynomial model:

$$
v=v_{f}\left(1-\left(\frac{\rho}{\rho_{J}}\right)^{n}\right)
$$

where $v_{f}$ - the free-flow speed; $\rho_{J}$ - the jammed density. $n=1$ is the Geenshields model (Geenshields , 1934). This model can be considered as a special case of the model proposed in this paper in Section 3.

(3) An exponential model used in (Hegyi et al, 2002):

$$
V(\rho)=v_{f} \exp \left(-\frac{1}{a}\left(\frac{\rho}{\rho_{c}}\right)^{a}\right)
$$

$v_{f}-$ free-flow speed;

$a-$ model parameter;

$\rho_{c}-$ critical density, the same as the $\rho_{m}$ used above.

It generalizes somehow the Underwood model.

(4) Some other models such as BPR model and Van Aerde model used in planning are referred to Skabardonis and Dowling (1997) and Van Aerde (1995).

\section{GENERALIZED POLYNOMIAL MODEL FOR $v(\rho)$}

Since the NGSIM data is microscopic from video camera instead of inductive loops, vehicle count and distance mean speed can be estimated directly. The $v-\rho$ relationship $v(\rho)$ is investigated. A new Generalized Polynomial model with non-integer power for FD is proposed for $v(\rho)$ with unit-sum and non-negative constraints on the coefficients.

\subsection{A Generalized Polynomial FD Model}

It can be shown that the most of the previous models can be approximated by or generalized to the following polynomial with non-negative coefficients and non-integer power:

$$
\begin{aligned}
& v(\rho)=V_{m} \cdot\left[1-\sum_{i=0}^{N} a_{i}\left(\frac{\rho}{\rho_{J}}\right)^{b_{i}}\right] \\
& b_{i}>0, a_{i} \geq 0, i=0,1, \ldots, N
\end{aligned}
$$

To avoid any ambiguity, $b_{i}, i=1, \ldots, N$ are assumed to be known real numbers and $b_{i} \neq b_{j}$, for $i \neq j$. (3.1) is called Generalized Polynomial FD Model. The nonnegative coefficients $\left(a_{0}, a_{1}, \ldots, a_{N}\right)$ are to be determined by fitting from practical data. In practice, one could choose lower or higher order instead of 6 .

\subsection{Concavity and Boundary Conditions}

Concavity is an important property for FD for the following reasons: (a) modelling of the static relationship; (b) understanding average driver behaviour; and (c) its potential application in traffic control. The concavity holding for previous models is also true for the Generalized Polynomial FD model (3.1) in $q-\rho$ relationship for $\rho \in\left(0, \rho_{J}\right]$. In fact, it is easy to calculate that

$$
\frac{d^{2} q(\rho)}{d \rho^{2}}<0 \text { for } \rho>0
$$

which means that $q(\rho)=\rho \cdot V(\rho)$ is strictly concave for $\rho>0$ since the coefficients $\left(a_{0}, a_{1}, \ldots, a_{N}\right)$ are all nonnegative. It is clear that $v(0)=v_{f}$ the free-flow speed. $v\left(\rho_{J}\right)=0$ leads to the constraint that $\sum_{i=0}^{N} a_{i}=1$. It is thus called Generalized Polynomial Model with Unit Sum Coefficients (GPMUSC). Intuitively, this model is generalization of previous models $(2.1-2.5)$ if one considers Taylor series approximation to order $N$.

Theorem 1. The generalized polynomial model (3.1) for the flow-density $(q-\rho)$ relationship is strictly concave for $\rho>0$.

\subsection{A Specific Model for $v(\rho)$}

In practice, (3.1) may be too general. A special case of the Generalized Polynomial FD Model is proposed. It is noted that $\frac{\rho}{\rho_{J}}<1$ for most interested cases, the power $\beta$ plays a significant role in $\left(\frac{\rho}{\rho_{J}}\right)^{\beta}, \beta>0$ since

$$
\begin{aligned}
& \left(\frac{\rho}{\rho_{J}}\right)^{\beta}>\frac{\rho}{\rho_{J}}, \beta<1 \\
& \left(\frac{\rho}{\rho_{J}}\right)^{\beta}<\frac{\rho}{\rho_{J}}, \beta>1
\end{aligned}
$$

To exploit such characteristics for modelling the traffic variation in transition phases, it might be necessary to include terms with non-integer power. Based on this consideration, the following model is proposed for $v(\rho)$ with the exponents set to some known values:

$$
\begin{aligned}
& V(\rho)=v_{m} \cdot\left(1-P\left(\frac{\rho}{\rho_{J}}\right)\right) \\
& P\left(\frac{\rho}{\rho_{J}}\right)=a_{0.3}\left(\frac{\rho}{\rho_{J}}\right)^{0.3}+a_{0.6}\left(\frac{\rho}{\rho_{J}}\right)^{0.6}+a_{1}\left(\frac{\rho}{\rho_{J}}\right)+a_{2}\left(\frac{\rho}{\rho_{J}}\right)^{2}+a_{3}\left(\frac{\rho}{\rho_{J}}\right)^{3}+a_{4}\left(\frac{\rho}{\rho_{J}}\right)^{4} \\
& a_{0.3}+a_{0.6}+a_{1}+a_{2}+a_{3}+a_{4}=1 \\
& a_{0.3} \geq 0, a_{0.6} \geq 0, a_{1} \geq 0, a_{2} \geq 0, a_{3} \geq 0, a_{4} \geq 0
\end{aligned}
$$


Linear Least Squares Method with non-negativity constraints (Lawson and Hanson, 1974) can be used for model fitting. For model calibration, one parameter is to be eliminated using the unit sum constraint, say, $a_{1}$ with

$$
\begin{gathered}
a_{1}=1-\left(a_{0.3}+a_{0.6}+a_{2}+a_{3}+a_{4}\right) \\
1-\frac{V}{v_{m}}=a_{0.3}\left[\left(\frac{\rho}{\rho_{J}}\right)^{0.3}-\left(\frac{\rho}{\rho_{J}}\right)\right]+a_{0.6}\left[\left(\frac{\rho}{\rho_{J}}\right)^{0.6}-\left(\frac{\rho}{\rho_{J}}\right)\right]+a_{2}\left[\left(\frac{\rho}{\rho_{J}}\right)^{2}-\left(\frac{\rho}{\rho_{J}}\right)\right]+ \\
a_{3}\left[\left(\frac{\rho}{\rho_{J}}\right)^{3}-\left(\frac{\rho}{\rho_{J}}\right)\right]+a_{4}\left[\left(\frac{\rho}{\rho_{J}}\right)^{4}-\left(\frac{\rho}{\rho_{J}}\right)\right] \\
a_{0.3} \geq 0, a_{0.6} \geq 0, a_{2} \geq 0, a_{3} \geq 0, a_{4} \geq 0
\end{gathered}
$$

which can be estimated using Least Square Method. It is noted that the proposed model generalizes several existing models based on Taylor expansion. It opens a new class of possible models for FD while keeping the concavity of $q-\rho$ relationship.

\section{VARIABLE STRUCTURE MODELING}

This section presents some variable structure models for the reversed $\lambda$ shape FD. The traffic drops from homogeneous flow happens between the two limbs. Edie model, and the GPMUSC are used to fit the left and right limbs of the reversed $\lambda$ shape FD.

\subsection{Reversed $\lambda$ Shape FD}

The simplest reversed $\lambda$ shape FD is to adopt straight line segment for the two limbs as in Figure 1.

Reversed $\lambda$ Shape FD

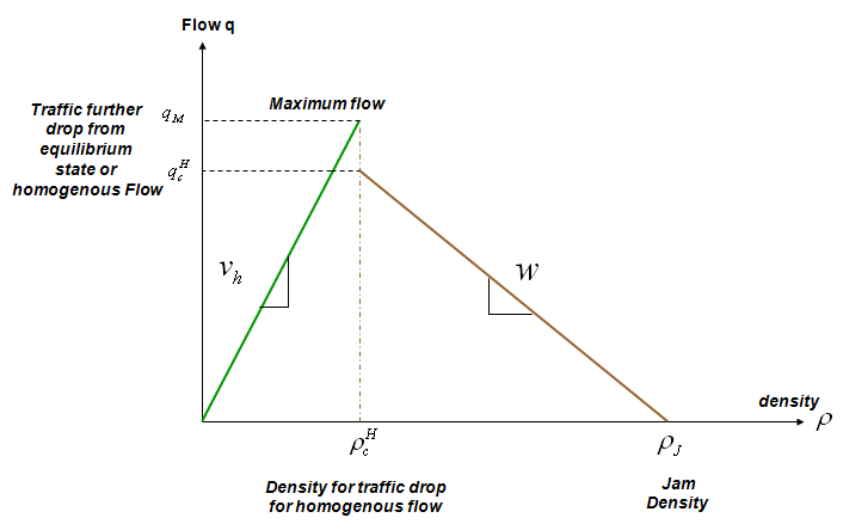

Figure 1. Reversed $\lambda$ Shape FD with both limbs as straight line (constant slope)

With this shape, the slope of the left limb is the homogenous-flow speed $v_{h}$. Several possible models for the left and right limbs are listed bellow.
(1) Linear model:

(2) Parabolic model:

$$
q=a_{1}+b_{1} \rho, \rho \leq \rho_{c}^{h}
$$

Right Limb Models:

$$
q=\alpha+\beta \rho+\gamma \rho^{2}, \quad \rho \leq \rho_{c}^{h}
$$

(1) Linear model:

$$
q=a_{2}+b_{2} \rho, \rho_{c}^{h}<\rho \leq \rho_{J}
$$

(2) GPMUSC Model for $\rho_{c}^{h}<\rho \leq \rho_{J}$ as in (3.2);

(3) Edie model for $\rho_{c}^{h}<\rho \leq \rho_{J}$ as in (2.3);

The combinations of the left and right limb with the above alternatives provides 6 models.

\subsection{Data for Model Fitting}

NGSIM data are used to fit them. Since current NGSIM data were collected in peak periods for congested traffic, it was not for a free-flow breakdown directly. The model calibration will focus on saturated traffic due to data availability. Since the data need to contain homogenousflow (not necessarily free-flow) traffic as well as shockwave, the data set for US101 Lane 1 in 3 time intervals are used including that for Figure 2. The distance aggregation is $170 \mathrm{~m}$ and time aggregation is $10 \mathrm{~s}$ which determines the size of the box A (aggregation level in time and space) in Figure 2.

Several time and distance aggregation intervals have tested in aggregating the vehicle-by-vehicle NGSIM data. Certainly, longer time and distance intervals for aggregation will generate neater static relationship but will swing further away from real-time control perspective. The most important criteria for the selection of time and distance intervals are as follows: (a) as short time and distance interval as possible to reduce time delay; (b) but they need to be appropriately long such that each interval has adequate data points so that traffic state parameters $(\rho, v, q)$ are well defined; (c) noise level is acceptable; and (d) the macroscopic relationship between traffic state parameters hold: $q=\rho v$. Based on those principles, the following aggregation intervals are suggested:

- time aggregation interval: $10 \sim 20 \mathrm{~s}$

- distance aggregation interval: 150 200m

In those levels, distance mean speed, density and thus flow are well-defined and the noise reduction through aggregation is acceptable. Data aggregation over distance $\Delta D$ and time $\Delta t$ is equivalent to shrink all the trajectory points within the rectangular box A with sides $\Delta D$ and $\Delta t$ into one point as shown in Figure 2 with Data Source: NGSIM data an US-101; Time: 07:50am-08:05am; Lane 1.

It is noted that the original NGSIM data update rate is $10 \mathrm{~Hz}$. The basic parameters to be estimated are distance means speed $v$ and density $\rho$ for each lane. The lane-flow $q$ is calculated from the former. 
The data are aggregated within the rectangular box as follows: vehicle count in that box divided by distance is the density $\rho$; the average of speed trajectories corresponding to those distance trajectories within the box is the distance mean speed $v$; and the flow $q$ is then calculated as $q=\rho \cdot v$. It is noted that data in box $\mathbf{A}$ should reflect shockwave, and data in box $\mathbf{B}$ homogenous flow.

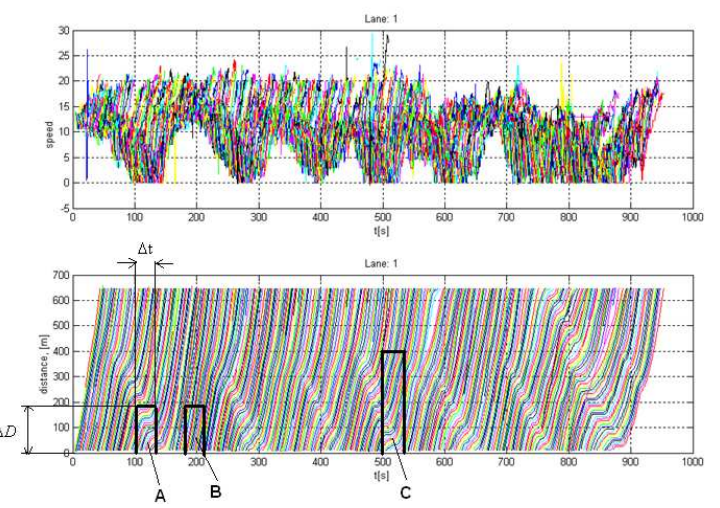

Figure 2. Upper/lower plot: individual vehicle speed /distance trajectories; distance trajectories were used for aggregation in a box with sides $\Delta D$ and $\Delta t$ to estimate traffic state parameters.

(1) Add the boundary condition under the assumption that $\rho_{J}=190 \mathrm{per} /$ lane $/ \mathrm{per} / \mathrm{mile}$ :

$$
v(0)=v(190)=0
$$

\subsection{Model Fitting and Observations}

The following facts are observed from model fitting using aggregated data:

(1) Break down density $\rho_{c}^{h}$ is in a range $\left[\rho_{c, 1}^{h}, \rho_{c, 2}^{h}\right]=[90,120]$. The value of $\rho_{c}^{h}$ which are different from lanes to lane;

(2) Selection of $\rho_{c}^{h}$ is important for two reasons:

a. if it is not selected properly, traffic breakdown at $\rho_{c}^{h}$ will not show up;

b. $\quad \rho_{c}^{h}$ selection affects estimation error for all the models with different magnitudes;

(3) In figure 3 and Figure $4, \rho_{c}^{h}$ is chosen such that the RMSE is minimum. Such a selection is possible because density has discrete value in the interval of interests;

(4) $\rho_{c}^{h}=95 \mathrm{veh} /$ lane-mile seems to be more reasonable than other choices;

\begin{tabular}{|l|l|l|l|l|l|}
\hline \multicolumn{5}{|c|}{ Data: US101 7:50 8:05am Lnl } \\
\hline \multicolumn{5}{|c|}{} & \multicolumn{3}{|c|}{ Right Limb Model Cofficients } \\
\hline $\begin{array}{l}\text { Left Limb } \\
\text { Model } \\
\text { Coefficien } \\
\text { ts }\end{array}$ & $\begin{array}{l}\text { Error } \\
\text { type }\end{array}$ & $\begin{array}{l}\text { GPMUSC } \\
v_{m}=38.6\end{array}$ & $\begin{array}{l}\text { Edie } \\
g_{1}=131.7 \\
g_{2}=-25.8\end{array}$ & $\begin{array}{l}\text { LIN } \\
a_{2}=2853.7 \\
b_{2}=-16.0\end{array}$ & \\
\hline $\begin{array}{l}\text { Linear: } \\
a_{1}=763.5 \\
b_{1}=12.6\end{array}$ & $v_{-}$err & 0.3448 & 0.3434 & 0.6010 & \\
\cline { 2 - 6 } & $q_{-}$err & 11.7049 & 11.2750 & 11.2121 & \\
\hline $\begin{array}{l}\text { Parabolic: } \\
\alpha=-608.5 \\
\beta=58.9 \\
\gamma=-0.4\end{array}$ & $v_{-}$err & 0.4821 & 0.4812 & 0.6890 & \\
\cline { 2 - 6 } & $q_{-}$err & 11.3106 & 10.8651 & 10.7997 & \\
\hline
\end{tabular}

It can be seen that for speed error, linear model for right limb is worse. However, for flow error, there is no significant different between the four models.

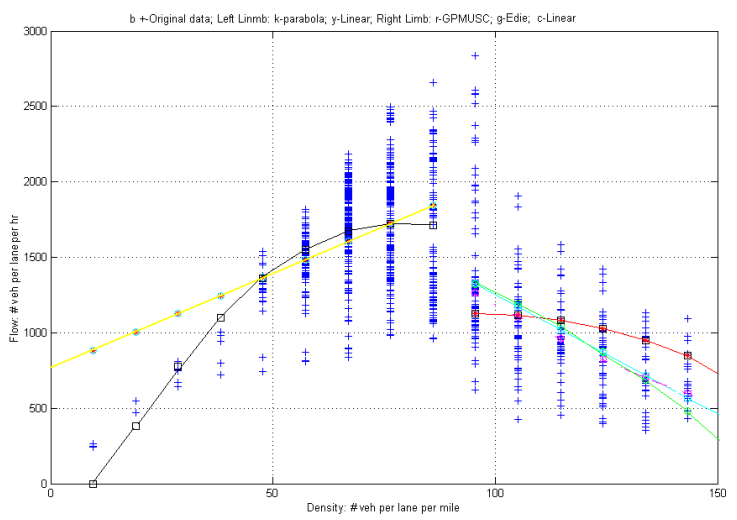

Figure 3. Variable Structure Model fitting from data set US101 7:50 8:05am Lane 1 in the first section of $170 \mathrm{~m}$; $\rho_{c}^{h}=95$ with time aggregation interval 10 steps (1s).

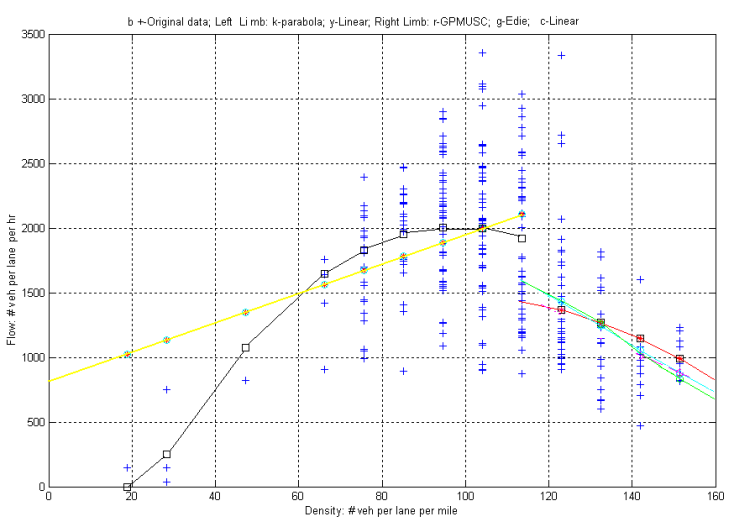

Figure 4. Variable Structure Model fitting from data set US101 Lane 1 and combined three time period: 07500805am, 0805-0820am, 0820-0835am; in the first section of $170 \mathrm{~m} ; \rho_{c}^{h}=115$ with time aggregation interval $10 \mathrm{~s}$; 


\begin{tabular}{|l|l|l|l|l|l|}
\hline \multicolumn{6}{|c|}{ Data: US101 7:50 8:05am Lnl } \\
\hline \multicolumn{2}{|c|}{} & \multicolumn{3}{|c|}{ Right Limb Model Coefficients } \\
\hline $\begin{array}{l}\text { Left Limb } \\
\begin{array}{l}\text { Model } \\
\text { Coefficien } \\
\text { s }\end{array}\end{array}$ & $\begin{array}{l}\text { Error } \\
\text { type }\end{array}$ & $\begin{array}{l}\text { GPMUSC } \\
\nu_{m}=38.6\end{array}$ & $\begin{array}{l}\text { Edie } \\
g_{1}=131.7 \\
g_{2}=-25.8\end{array}$ & $\begin{array}{l}\text { LIN } \\
a_{2}=2853.7 \\
b_{2}=-16.0\end{array}$ & \\
\hline $\begin{array}{l}\text { Linear: } \\
a_{1}=763.5 \\
b_{1}=12.6\end{array}$ & $v_{-}$err & 0.3448 & 0.3434 & 0.6010 & \\
\cline { 2 - 6 } & $q_{\text {err }}$ & 11.7049 & 11.2750 & 11.2121 & \\
\hline $\begin{array}{l}\text { Parabolic: } \\
\alpha=-608.5 \\
\beta=58.9\end{array}$ & $v_{-}$err & 0.4821 & 0.4812 & 0.6890 & \\
\cline { 2 - 6 } \\
$\gamma=-0.4$ & $q_{-}$err & 11.3106 & 10.8651 & 10.7997 & \\
\hline
\end{tabular}

Similarly, for speed estimation error, linear model for right limb is worse than other three. However, for flow estimation error, there is no significant difference between the four models. It can also be observed that using data from different time periods for the same location leads to different estimation error. This, together with the difference in model coefficients, suggests that FD might not be static in time.

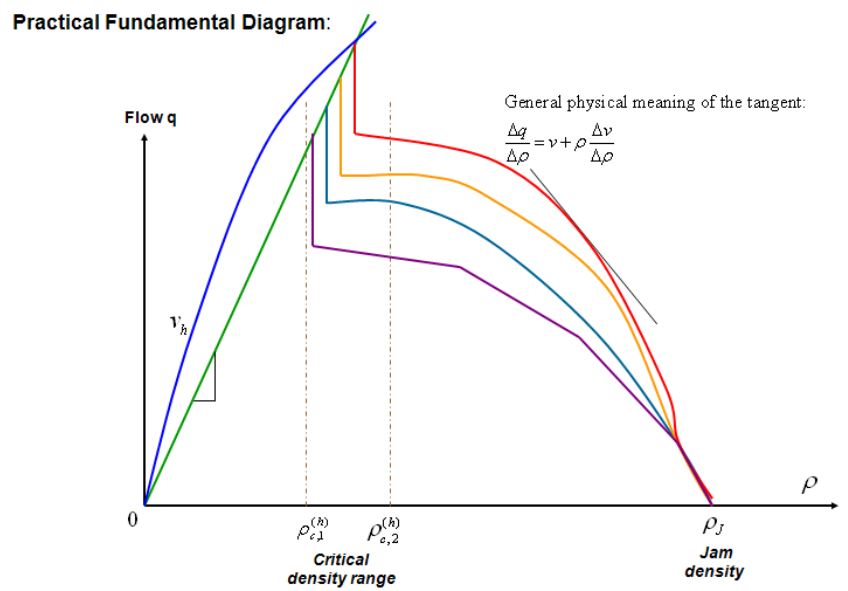

Figure 5. FD for homogenous flow; $v_{h}$ is the speed of the homogenous flow; breakdown density for a homogenous flow falls into a range $\left[\rho_{c, 1}^{h}, \rho_{c, 2}^{h}\right]$ which is different in time and locations.

\subsection{Practical Shape of FD}

Based on the model variable structure model fitting for the reversed $\lambda$ shape model, a general shape of a practical FD is proposed in Figure 5. With density in the $\operatorname{range}\left[\rho_{c, 1}^{h}, \rho_{c, 2}^{h}\right]$, the homogenous traffic would have higher probability to breakdown.

It would be interesting to analyze analytically the physical meaning of the tangent for the right limb of the FD curve (Figure 5). Starting from $q=v \cdot \rho$ and using the difference rule in mathematics, it is obtained that

$$
\begin{aligned}
& q=v \cdot \rho \\
& \Delta q=\Delta v \cdot \rho+v \cdot \Delta \rho \\
& \frac{\Delta q}{\Delta \rho}=v+\frac{\Delta v}{\Delta \rho} \cdot \rho
\end{aligned}
$$

It is clear to have the following:

General Physical Meaning for the Tangent of Right Limb in FD: the tangent in the right limb is the distance means speed $v$ plus an increment term $\frac{\Delta v}{\Delta \rho} \rho$ which must be negative since $\frac{\Delta v}{\Delta \rho}<0$.

\section{CONCLUDING REMARKS}

FD describes the flow-density $q-\rho$ (speed-density $v-\rho$ ) relationship. FD is very important in understanding macroscopic traffic model and critical for traffic control such as ramp metering and VSL in model simplification, capacity estimation, and prediction of traffic drops from a homogenous flow, of which a special case is traffic direct breakdown from free-flow. This paper proposed a generalized polynomial model for the $v-\rho$ (or $q-\rho$ ) function which naturally satisfy the boundary conditions. Properly aggregated NGSIM data for saturated traffic are used for model calibration and comparison. Linear and parabolic lines are used for left limb; Edie, linear and GPMUSC are used for right limb. RMSE indicates that they are not significantly different for $q-\rho$ relationship although the GPMUSC is slightly better for $v-\rho$ relationship. The linear mode is not as good for the right limb of $v-\rho$. It thus opens a new class of models for further investigation.

Proper length of the distance and time intervals used for data aggregation from microscopic data to macroscopic data are very recognized important. Too short distance interval will not generate meaningful traffic parameters such as density, distance mean speed and flow $(\rho, v, q)$; too long distance would smooth out traffic characteristics such as shockwaves and flow-drops. The following principles are proposed for traffic data aggregation: (a) as short time and distance interval as possible to reduce time delay (for traffic control purpose); (b) but they need to be long enough such that each interval has adequate data points so that traffic state parameters $(\rho, v, q)$ are well defined; (c) noise level is acceptable; and (d) the macroscopic relationship between traffic state parameters hold: $q=\rho v$. Obvious, there is trade-off in the selection of such intervals in practice.

For NGSIM (peak hour traffic) vehicle-by-vehicle tracking data with $10 \mathrm{~Hz}$ update rate, the distance interval should be between $150 \sim 200 \mathrm{~m}$ and time interval be 10 20s. 
Different model coefficients determined through Least Squares fitting from data at the same location but different time periods and the same time periods but different locations indicate that the FD is neither static in time nor homogenous in distance. This is also enhanced by the fact that the estimation errors are different for the same model but data from different locations of the same time period, or the same location but different time periods. How those results would mean for traffic modelling and control needs further consideration.

All the static models only represent some static relationships between speed (flow) and density. It can only represent the traffic in an equilibrium state. Even in this case, data has to be properly aggregated over time for fixed location to induce such a relationship. Thus the use of use of FD must be under certain conditions. However, FD reflects to some extent the driver behaviour which could be incorporated in dynamic model in the future. The eventual purpose is to use some aspects of the FD in traffic control design as well be addressed in the future.

\section{ACKNOWLEDGEMENT}

This work was supported by the Federal Highway Administration (FHWA) Exploratory Advanced Research Project (Cooperative Agreement DTFH61-07-H-00038) with Match Funding from the California Department of Transportation (Caltrans) TO6224. The contents of this report reflect the views of the authors who are responsible for the facts and the accuracy of the data presented herein. The contents do not necessarily reflect the official views or policies of FHWA and the State of California. This paper does not constitute a standard, specification, or regulation.

\section{REFERENCES}

Alexiadis, V., Colyar, J. Halkias, J., Hranac, R., and McHale, G. (2004). The Next Generation Simulation Program. ITE Journal, Vol. 74, No. 8, p. 22-26.

Del Castillo, J.M. and Benitez, F.G., (1995). On the functional form of the speed-density relationship. Part Two: Empirical investigation. Transportation Research B 29, 391-406.

Daganzo, C. F. (1994). The Cell Transmission Model: A Dynamic Representation of Highway Traffic Consistent with the Hydrodynamic Theory. Transportation Research -B, Vol. 28, No. 4, 1994, pp. 269-287.

Daganzo, C. F. (1995). The Cell Transmission Model, Part II: Network Traffic. Transportation Research -B, Vol. 29, No. 2, pp. 79-93.

Edie, L. C., Car following and steady state theory for noncongested traffic, Operations Research, 9, (1961), p66-76

Greeberg, H., (1959). An analysis of traffic flow, Operations Research, Vol. 7, no. 1, p79-85

Greenshields, B. D., (1934). A study of traffic capacity, HRB Proc. 14, p448-481

Hegyi, A.; De Schutter, B.; Hellendoorn, H.; van den Boom, T., (2002). Optimal coordination of ramp metering and variable speed control-an MPC approach, Proc. American Control Conf. Vol. 5, p3600-3605

Lighthill M. J. and Whitham G. B. (1955) On kinematic waves II. A theory of traffic flow on long crowded roads. Proc. Royal Society of London, Series A, 229, 317-345.

Lawson, C.L. and R.J. Hanson, (1974). Solving Least Squares Problems, Prentice-Hall, Chapter 23, p. 161.

Lu, X. Y. and Skabardonis, A., Freeway Traffic Shockwave Analysis: Exploring the NGSIM Trajectory Data, 86th TRB Annual Meeting, Washington, D.C.,Jan. 21-25, 2007

Underwood, R. T., (1961). Speed, volume and density relationships, Quality and Theory of Traffic Flow, Yale Bureau of Highway Traffic, p141-88

Van Aerde, M. (1995). Single regime speed-flow-density relationship for congested and uncongested highways. 74th TRB Annual Conference, Washington D.C., Paper No. 95080.

Skabardonis, A. and Dowling, R., (1997), Improved speedflow relationships for planning applications, Transportation Research Record, \#1572

Zhang, H.M., (1999). A mathematical theory of traffic hysteresis. Transportation Research, B 33, p1-23 